\title{
Case Report: Progression of Pre-Septal Cellulitis to Orbital Subperiosteal Abscess despite Intravenous Broad-Spectrum Antibiotics in a Child
}

\author{
Noor Aniah $\mathrm{A}^{1,2}(\bowtie)$, Norshamsiah $\mathrm{MD}^{1}$, Bastion MLC ${ }^{1}$, Safinaz $\mathrm{MK}^{1}$, Mawaddah $\mathrm{A}^{3}$ \\ ${ }^{1}$ Department of Ophthalmology, ${ }^{3}$ Department of Otorhinolaryngology Faculty of Medicine, Universiti \\ Kebangsaan Malaysia Medical Centre, Jalan Yaacob Latif, Bandar Tun Razak, 56000 Cheras, Kuala \\ Lumpur, Malaysia. \\ ${ }^{2}$ Ophthalmology Unit, Department of Surgery, Medical and Health Science Faculty, Universiti Putra \\ Malaysia, 43400 UPM Serdang, Selangor Darul Ehsan, Malaysia.
}

\begin{abstract}
We report a case of a 7-year-old girl who initially presented with painless right eyelid swelling with full extra-ocular movement (EOM). She was treated with intravenous broad-spectrum antibiotics for preseptal cellulitis but her condition worsened. An urgent magnetic resonance imaging (MRI) of the brain and orbit showed orbital abscess, subperiosteal abscess in the medial orbital wall and evidence of sinusitis in the anterior ethmoidal air cells. She underwent Endoscopic Orbital Decompression (EOD) surgery on day 4 of presentation and her condition improved remarkably. We report a case of orbital abscess with subperiosteal abscess in the medial orbital wall. This case highlights the possibility of progression of orbital cellulitis despite administration of a broad-spectrum antibiotic.
\end{abstract}

Keywords: $\quad$ case reports, orbital abscess, orbital cellulitis, paediatric, subperiosteal abscess

\section{Correspondence:}

Noor Aniah Azmi, Department of Ophthalmology, Faculty of Medicine, Universiti Kebangsaan Malaysia Medical Centre, Jalan Yaacob Latif, Bandar Tun Razak, 56000 Cheras, Kuala Lumpur, Malaysia. Tel: +603-9145 7207 Fax: +603-9145 6733 Email: nooraniah@gmail.com

\section{Introduction}

Orbital cellulitis is a sight-threatening orbital inflammation, which can cause fatal complications to the central nervous system. Prior to development of antibiotics, $17 \%$ of patients died from the complication of meningitis while $20 \%$ of cases went blind (1). The incidence of orbital cellulitis was reported to be 1.6 in 100,000 children below 17 years of age (2). Common initial presentation includes eyelid swelling, restricted eye movement and proptosis. We report a case of painless preseptal cellulitis, which progressed to orbital cellulitis despite intravenous antibiotics.

\section{Case Report}

A 7-year-old Caucasian girl presented with painless right erythematous eyelid swelling for one day prior to presentation to another ophthalmology unit. Examination revealed good visual acuity, no relative afferent pupillary defect (RAPD), no proptosis or limitation in eye movement and no report of diplopia. The conjunctiva was white and she was afebrile in the ward. She had a history of viral fever one week prior to presentation with complaint of cough and runny nose. She was treated as preseptal cellulitis with intravenous Ceftriaxone $(50 \mathrm{mg} / \mathrm{kg} /$ day $)$. The eyelid swelling improved after two doses of intravenous Ceftriaxone. However, three days later while still in the ward, she complained of severe right eye pain especially on down gaze. Her best-corrected visual acuity was 6/9, N5 in both eyes. The extraocular motility of the right eye was very limited due to severe pain. The anterior and posterior segment examination was normal. Her intraocular pressure (IOP) was $14 \mathrm{mmHg}$ in both eyes. Exophthalmometer was not performed as the patient was 


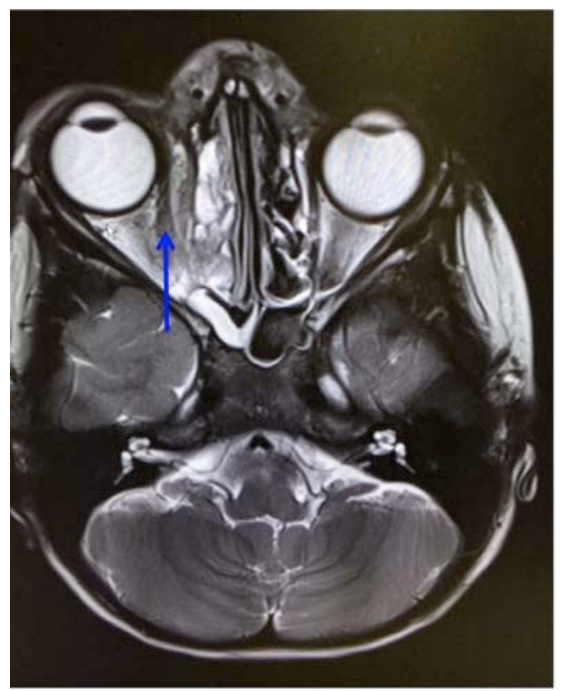

Figure 1: Axial T2-weighted MRI on day 4 of onset showing collection of pus in the orbit and subperiosteal space of the medial orbital wall (blue arrow) with collection in the right ethmoidal sinus

not cooperative. Her vital signs were normal with no documented pyrexia. Her blood investigations showed raised white cell count of $15.3 \times 103 / \mu \mathrm{L}$ with predominant neutrophils (75\%). An urgent MRI of the orbit and brain revealed right eye proptosis, right orbital and subperiosteal abscess involving the medial wall with pus collection in the paranasal sinuses (ethmoid, sphenoid and maxillary) (Fig. 1). She was then referred to our ophthalmology and otorhinolaryngology units for further management. She underwent emergency Endoscopic Orbital Decompression (EOD) under general anaesthesia. Intraoperatively, there was thick purulent collection in the paranasal sinuses and upon opening the lamina papyracea confirming the clinical diagnosis of acute sinusitis complicated with subperiosteal abscess. The culture and sensitivity of the pus showed no growth after 72 hours. She made tremendous recovery postoperatively. (Fig. 2a \& 2b). She did not develop re-accumulation of pus or other complications from the surgery. Postoperatively, she was given intravenous Ceftriaxone $500 \mathrm{mg} \mathrm{BD}$ and Metronidazole $150 \mathrm{mg}$ TDS. On her last follow-up 1 month following the surgery, examination revealed full EOM, Hess chart and binocular single vision chart were normal.

\section{Discussion}

Orbital abscess is a known complication of orbital cellulitis whereby pus collects within the orbital soft tissues, while subperiosteal abscess denotes collection of pus between the bones of the orbit and the periosteum. Ferguson et al reported that $62 \%$ of paediatric

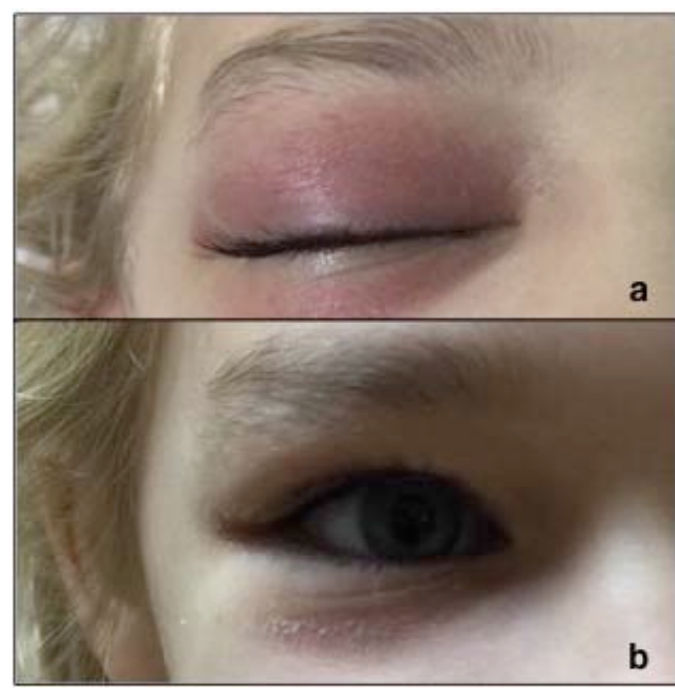

Figure 2: Coloured photograph of the patient a) prior to EOD drainage showing erythematous eyelid swelling and inability to open the eye at rest, b) day 2 post-EOD drainage showing improvement of the eyelid swelling.

orbital cellulitis have underlying subperiosteal abscess (SPA) (3). In orbital abscess and SPA, the infection extends beyond the orbital septum. The orbital septum is a sheet of connective tissue that acts as a tough barrier to prevent spread of facial infection into the orbit as well as keeping the orbital contents in place. Chandler graded orbital cellulitis into five grades according to disease severity. Group I is preseptal cellulitis, group II is orbital cellulitis, group III is SPA, group IV is orbital abscess and group $\mathrm{V}$ is cavernous sinus thrombosis. These groups do not necessarily imply the sequence of disease progression. The most common source of infection in orbital cellulitis is from the adjacent paranasal sinuses, where about 33\% of them are from the maxillary and ethmoidal sinuses (4) because of the thin, fenestrated walls of the ethmoid sinus which acts as a poor barrier. About $88 \%$ of SPA involves the medial orbital wall as it is close to the paranasal sinuses (5).

Acute sinusitis in children is usually preceded by a viral infection causing swelling of the nasal mucosa and narrowing of the sinus ostia. This favours a secondary infection particularly aerobic infection followed by a mixed aerobic and anaerobic infection (6). Children are more susceptible to develop orbital cellulitis from sinusitis due to smaller airway passages and their immune-compromised state. In children, Streptococi species and Haemophilus influenza are yielded in $70 \%$ of cases (2). However, in our patient, the culture and sensitivity showed no growth after 72 hours as she has been partially-treated with intravenous Ceftriaxone. In cases of anaerobic 
infection, Ceftriaxone and Metronidazole are administered typically concurrently to provide a broader spectrum of coverage (7). Anaerobic infection should suspected in cases of dental sinusitis, rapid progression of signs, diabetic patients and foulsmelling nasal discharge. If this is suspected, Metronidazole should be added. On the other hand, Ceftriaxone which is a third-generation cephalosporins provides good coverage for Hemophilus Influenza. In SPA, there is a possibility of microbial resistance such as methicillin-resistant StaphylococusAureus (MRSA) infection. An antimicrobial agent with good penetration to the central nervous system like Clindamycin or Vancomycin is usually added (8). In this case, we chose to add Metronidazole due to its less systemic toxicities and the convinience of antibiotic therapy as outpatient.

Post-septal orbital infections usually present with painful eyelid swelling, proptosis, decreased vision and systemic toxicity. Majority of paediatric patients present with ophthalmoloplegia (71\%), proptosis (76\%) and fever (71\%), while only 32\% will present with reduced visual acuity. Early features of orbital abscess are pain on eye movement before development of reduced vision, especially in SPA where the extraocular muscle (EOM) is closer to the subperiosteum (3). The cause of decreased vision in orbital cellulitis could be due to optic neuritis, or from mechanical pressure of the abscess onto the arteries supplying the optic nerve and causing direct increase in IOP (1).

In a case series reported by Harris et al, the patients who were diagnosed with SPA presented with erythematous eyelid swelling with limited EOM at initial presentation $(9,10,11)$. In contrast to our patient, she developed eye pain and limited EOM only after 3 days of intravenous antibiotics. It was reported that medial orbital wall SPA from a non-dental source in children younger than 9 years old respond well to medical treatment (12). However, the present case required surgical intervention despite initial good response towards medical treatment. The fact that her intraoperative pus sample taken from the subperiosteal abscess revealed no growth could indicate that there is initial good drug penetration and with further accumulation, penetration is impaired. We postulate that her deterioration is due to ongoing development and accumulation of pus as the subperiosteum is a potential space. Delayed surgical intervention can further worsen her condition because the subperiosteum is relatively avascular where access of drug and host immune response may not be favourable (6). The inflammatory response can impact the extraocular muscles which causes the patient to have pain on eye movement.
In SPA for children older than 9 years old, many ophthalmologists advocate for surgical drainage, as there are reported cases where they do not respond to medical treatment (13). The reason is because of the multiplicity of pathogens yielded which makes medical management challenging. The indication of surgical drainage in SPA includes marked orbital symptoms, worsening condition after 24 hours of medical treatment or failure of treatment after 48 hours (8). Smith et al. suggested for surgical drainage in cases with risk factors of EOM restriction, proptosis, age more than 9 years old and increase IOP (12). The volume and degree of exophthalmometer is an important indicator whether the patient should undergo surgical drainage (14). It was suggested that proptosis of more than $2 \mathrm{~mm}$ should be considered as a factor for surgical drainage. In the present case, the patient's proptosis level was not recorded. EOD is highly recommended in drainage of SPA as it is a minimallyinvasive procedure with shortened hospital stay but requires a highly-skilled surgeon to perform especially in the paediatric age group (15).

Differentiation of preseptal cellulitis and orbital cellulitis is crucial as the latter is associated with morbidity and mortality. The ideal method of recognizing this diagnosis is via computed tomography (CT), although it is not a routine investigation in preseptal cellulitis in the paediatric age group due to risks of radiation exposure. CT scan is favoured because it can determine the type and extent of the condition. SPA appears as a hypodense lesion with ring enhancement along the periosteum on CT scan while orbital abscess appears as collection of pus in the orbital region. MRI and ultrasound of the orbit may also differentiate between preseptal and orbital cellulitis however, it is a lengthy procedure that may not be suitable in uncooperative paediatric patients. (16). However, ultrasound may not be able to detect involvement of the orbital apex. A sinus palpation may exclude sinusitis which is closely-related to orbital cellulitis but again may not be reliable in paediatric patients.

\section{Conclusion}

Paediatric patients who present with eyelid swelling with no evidence of orbital involvement or abscess should be admitted and receive intravenous broadspectrum antibiotics for a mimimum of 3 days. Daily monitoring for progression to orbital abscess is warranted as paediatric patients may still progress to subperiosteal abscess, which requires surgical drainage despite commencement of antibiotics. Hence cases of this type warrant co-management with the otorhinolaryngology team. CT scan of the orbit and 
paranasal sinuses should be performed when there is clinical suspicion or evidence of new symptoms and signs to suggest progression to orbital cellulitis and orbital abscess. Early surgical drainage will avoid a vision-threatening and life-threatening condition especially in the pediatric age group.

\section{References}

1. Chaudhry IA, Al-Rashed W, Arat YO. The hot orbit: Orbital cellulitis. Middle East Afr J Ophthalmol 2012; 19(1): 34-42.

2. Murphy C, Livingstone I, Foot B, Murgatroyd H, MacEwen CJ. Orbital cellulitis in Scotland: current incidence, aetiology, management and outcomes. Br J Ophthalmol 2014; 98(11): 15758.

3. Ferguson MP, McNab AA. Current treatment and outcome in orbital cellulitis. Aust New Zeal J Ophtalmol 1999; 27(6): 375-9.

4. Aldemir Kocabas B, Karbuz A, Ozdemir H, Ciftci E, Ince E. Periorbital and orbital cellulitis: from presentation to outcome. Çocuk Enfeksiyon Dergisi/J Pediatr Inf 2014; 8(4): 148-52.

5. Mathew AV, Craig E, Al-Mahmoud R, et al. Paediatric post-septal and pre-septal cellulitis: 10 years' experience at a tertiary-level children's hospital. Br J Radiol 2014; 87(1033): 20130503.

6. Harris GJ. Age as a factor in the bacteriology and response to treatment of subperiosteal abscess of the orbit. Trans Am Ophthalmol Soc 1993; 91: 441-516.

7. Laffont J, Augusto S, Cardoso L, Ribeiro JC, Paiva A. Orbital subperiosteal abscess at pediatric age. Ann Otolaryngol Rhinol 2015; 2(3): 1029.
8. Bedwell J, Bauman NM. Management of pediatric orbital cellulitis and abscess. Curr Opin Otolaryngol Head Neck Surg 2011; 19(6): 467-73.

9. Harris GJ. Subperiosteal abscess of the orbit. Arch Ophthalmol. 1983; 101(5): 751-7.

10. Blumenthal DS, Powell O. Orbital cellulitis and sinusitis caused by group B beta streptococcus in a 3-year-old child. J Natl Med Assoc 1985; 77(2): 147-8.

11. de Medeiros EH, Pepato AO, Sverzut CE, Trivellato AE. Orbital abscess during endodontic treatment $\square$ : a case report. J Endod 2012; 38(11): 1541-3.

12. Smith JM, Bratton EM, DeWitt P, Davies BW, Hink EM, Durairaj VD. Predicting the need for surgical intervention in pediatric orbital cellulitis. Am J Ophthalmol 2014; 158(2): 387-94.e1.

13. Vairaktaris E, Moschos MM, Vassiliou S, et al. Orbital cellulitis, orbital subperiosteal and intraorbital abscess: report of three cases and review of the literature. J Craniomaxillofac Surg 2009; 37(3): 132-6.

14. Tabarino $F$, Elmaleh-Bergès $M$, Quesnel $S$, Lorrot M, Van Den Abbeele T, Teissier N. Subperiosteal orbital abscess: volumetric criteria for surgical drainage. Int $\mathrm{J}$ Pediatr Otorhinolaryngol 2015; 79(2): 131-5.

15. Deutsch E, Eilon A, Hevron I, Hurvitz H, Blinder G. Functional endoscopie sinus surgery of orbital subperiosteal abscess in children. Int J Pediatr Otorhinolaryngol 1996; 34(1-2): 181-90.

16. Derr C, Shah A. Bedside ultrasound in the diagnosis of orbital cellulitis and orbital abscess. Emerg Radiol 2012; 19(3): 265-7. 